\author{
U. P. Mosimann - J. Felblinger $\cdot$ S. J. Colloby $\cdot$ \\ R. M. Müri
}

\title{
Verbal instructions and top-down saccade control
}

Received: 16 March 2004 / Accepted: 27 July 2004 / Published online: 2 October 2004

(C) Springer-Verlag 2004

\begin{abstract}
Few studies have addressed the interaction between instruction content and saccadic eye movement control. To assess the impact of instructions on top-down control, we instructed 20 healthy volunteers to deliberately delay saccade triggering, to make inaccurate saccades or to redirect saccades-i.e. to glimpse towards and then immediately opposite to the target. Regular pro- and antisaccade tasks were used for comparison. Bottom-up visual input remained unchanged and was a gap paradigm for all instructions. In the inaccuracy and delay tasks, both latencies and accuracies were detrimentally impaired by either type of instruction and the variability of latency and accuracy was increased. The intersaccadic interval (ISI) required to correct erroneous antisaccades was shorter than the ISI for instructed direction changes in the redirection task. The word-by-word instruction content interferes with top-down saccade control. Top-down control is a time consuming process, which may override bottom-up processing only during a limited time period. It is questionable whether parallel processing is possible in top-down control, since the long ISI for instructed direction changes suggests sequential planning.
\end{abstract}

Keywords Instruction $\cdot$ Saccade $\cdot$ Antisaccade $\cdot$ Topdown control $\cdot$ Bottom-up control

U. P. Mosimann · J. Felblinger · R. M. Müri

Perception and Eye Movement Laboratory, Departments of

Neurology and Clinical Research, University of Bern,

Bern, Switzerland

U. P. Mosimann $(\bowtie) \cdot$ S. J. Colloby

Institute for Ageing and Health, Newcastle General Hospital, Newcastle upon Tyne, NE4 6BE, UK

e-mail: u.p.mosimann@ncl.ac.uk

Tel: +44-191-2563018

Fax: +44-191-2195071

J. Felblinger

Department of Radiology, University of Nancy,

Nancy, France

\section{Introduction}

Saccadic eye movements are outside of conscious selfawareness, but all saccades, even so-called reflexive saccades can be volitionally controlled (Leigh and Kennard 2003). When saccades are tested in humans, verbal instructions usually provide the temporo-spatial information, and despite the knowledge that the instruction content interferes with saccade control, only very few studies have addressed the interaction of instruction and saccade control in humans (Fischer et al. 1993; Kowler and Blaser 1995). Instructions aim to optimise performance, but this approach is susceptible to ceiling or floor effects due to the physiological limitations in decreasing latency or increasing accuracy, when visual information is perceived. The verbal instruction content is not as crucial in animal studies, and since a lot of eye movement studies have been done in animals, this may be a reason for the lack of research in this area in humans. The general assumption that humans do their best to follow instructions is reasonable, although there are disorders where the internal instruction may differ from the external instruction (Turner 1997).

Top-down control can be applied to processing driven by expectation and volition and bottom-up control to processing driven autonomously by the incoming visual information. Cognitive and physiological models of saccade generation (Findlay and Walker 1999; Hikosaka et al. 2000; Pierrot-Deseilligny et al. 2003b) suggest an overlap between top-down and bottom-up control and assume that saccade latency, accuracy and choice of direction can be top-down controlled. In order to assess the interaction between instruction and top-down control, different instructions were given to healthy controls, while bottom-up visual input was a gap paradigm for all instructions. We instructed healthy volunteers to delay saccade triggering, to make inaccurate saccades and to redirect saccades. Regular pro- and antisaccades were used for comparison. Instructions to perform poorly were chosen to maximise top-down influences in order to get 
an optimal separation between top-down and bottom-up processing.

\section{Materials and methods}

Subjects

Twenty healthy volunteers ( 7 females, 13 males), mean age of 28.7 years (SD 6.1 years) were assessed. All subjects had normal visual acuity and none were on psychotropic medication or had a history of current or past psychiatric or neurological disorders. The local ethics committee approved the study and subjects gave informed consent.
Eye movement recordings

Eye movements were recorded with an infrared light reflection system (IRIS SKALAR) in a standardized environment in the Perception and Eye Movement Laboratory at University of Bern, Switzerland. The data was sampled at a frequency of $200 \mathrm{~Hz}$ and the digitised signal stored on a computer for offline analysis. Subjects were seated in complete darkness $1.2 \mathrm{~m}$ in front of a light emitting diodes (LED) screen. Their head was fixed on a combined chin and forehead-holder. Prior to each block the signal was calibrated. Each subject determined individually the number and length of breaks required, and the whole experiment lasted approximately $30 \mathrm{~min}$. All subjects were naïve to the goal of the study.

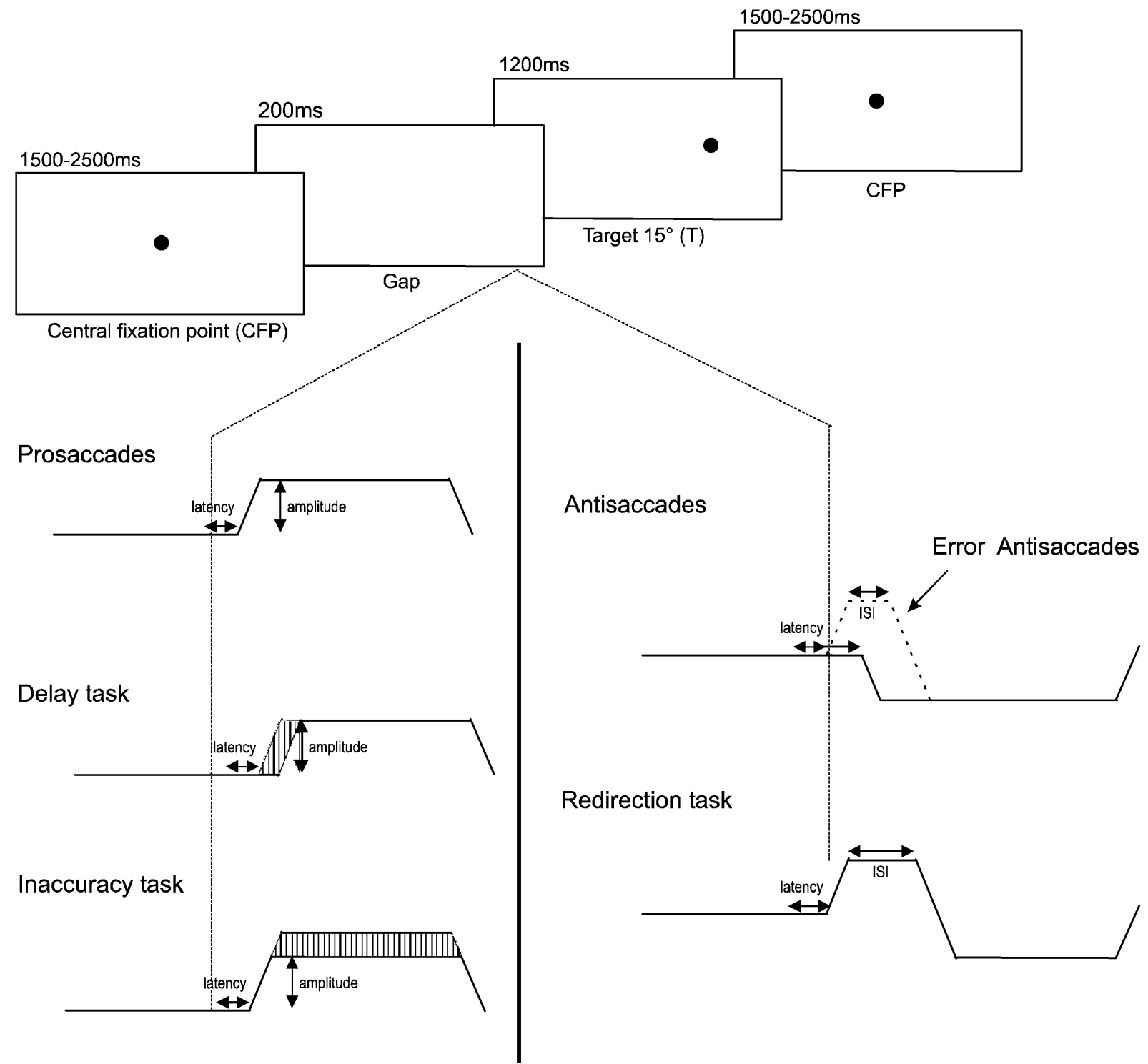

Fig. 1 This figure summarizes the stimulus sequence and the different tasks used in the present study. Stimulus sequence was a gap paradigm and the timing and directions of the stimuli were

unpredictable. The instructions were to delay saccade triggering, to be inaccurate, or to redirect saccades. Prosaccade and antisaccade tasks were used for comparison 
Stimulus sequence

The stimulus sequence was a gap paradigm for all instructions. Four blocks of 10 saccades were tested per instruction (i.e. 40 saccades per instruction). A central fixation point (CFP) was presented randomly for 1,500 $2,500 \mathrm{~ms}$. The CFP disappeared $200 \mathrm{~ms}$ before target onset and targets were presented for 1,200 ms (Fig. 1). Target direction and timing of target appearance were unpredictable and target eccentricity was 15 degrees. The present report is based on the measurement of 4,000 saccades (200 saccades per subject).

\section{Instructions}

All instructions were read to the subjects by the experimenter in a random order.

1. Prosaccade instruction: Please look at the targets as precisely and as fast as possible.

2. Delay instruction: Please delay looking at the targets, but be as precise as possible. Do not miss any targets.

3. Inaccuracy instruction: Please look as fast as possible but inaccurately towards the targets. Do not look opposite of the targets.

4. Antisaccade instruction: Please look as fast as possible to the opposite side of the targets.

5. Redirection instruction: Please glimpse towards, then immediately opposite to the targets.

\section{Outcome measures}

For the prosaccade-, delay- and inaccuracy tasks primary outcome measures were saccade latency and accuracy (i.e. gain $=$ saccade amplitude/target amplitude). To assess variability, SDs were compared. Prosaccade latency $(95 \%$ confidence interval, CI) was used to define delayed saccades in the delaying task: latencies above $95 \%$ CI of regular prosaccades were considered to be delayed. The same procedure was used for the gain in the inaccuracy task and saccades below 95\% CI of regular prosaccades were defined as hypometric, and those above as hypermetric. The intersaccadic interval (ISI), i.e. the time required for direction changes, was analysed in erroneous antisaccades compared with the ISI in the redirection task.

Data analysis and definitions

Anticipated saccades, i.e. saccades with latencies below 80 $\mathrm{ms}$ and saccades triggered after target disappearance (after $1,200 \mathrm{~ms}$ ) were rare (less than $5 \%$ of all saccades) and excluded from the analysis. The distribution of the data was tested for normality (Kolmogorov-Smirnov test) and since data were normally distributed, means, standard deviations (SD) and 95\% CI were reported and parametric tests (dependent sample $t$-tests) were applied. All reported $p$ values were two tailed and $p$ values of less than 0.05 were considered to be statistically significant.

\section{Results}

Figure 2 summarizes mean and $95 \% \mathrm{CI}$ of saccade latency (Fig. 2a) and gain (Fig. 2b) of the prosaccade, delay and inaccuracy tasks. Mean latency of delayed saccades was significantly longer than the latency of prosaccades $(p<0.001)$. Compared to regular prosaccades, delayed saccades were less accurate, i.e. had lower gain $(p=0.002)$. The SDs of latencies following delay instruction were higher compared to the SDs of prosaccade latencies $(p<0.0001)$ (Table 1). When instructed to delay saccade triggering, 22\% (SD 21\%) were not delayed but triggered within the CI of regular prosaccades.
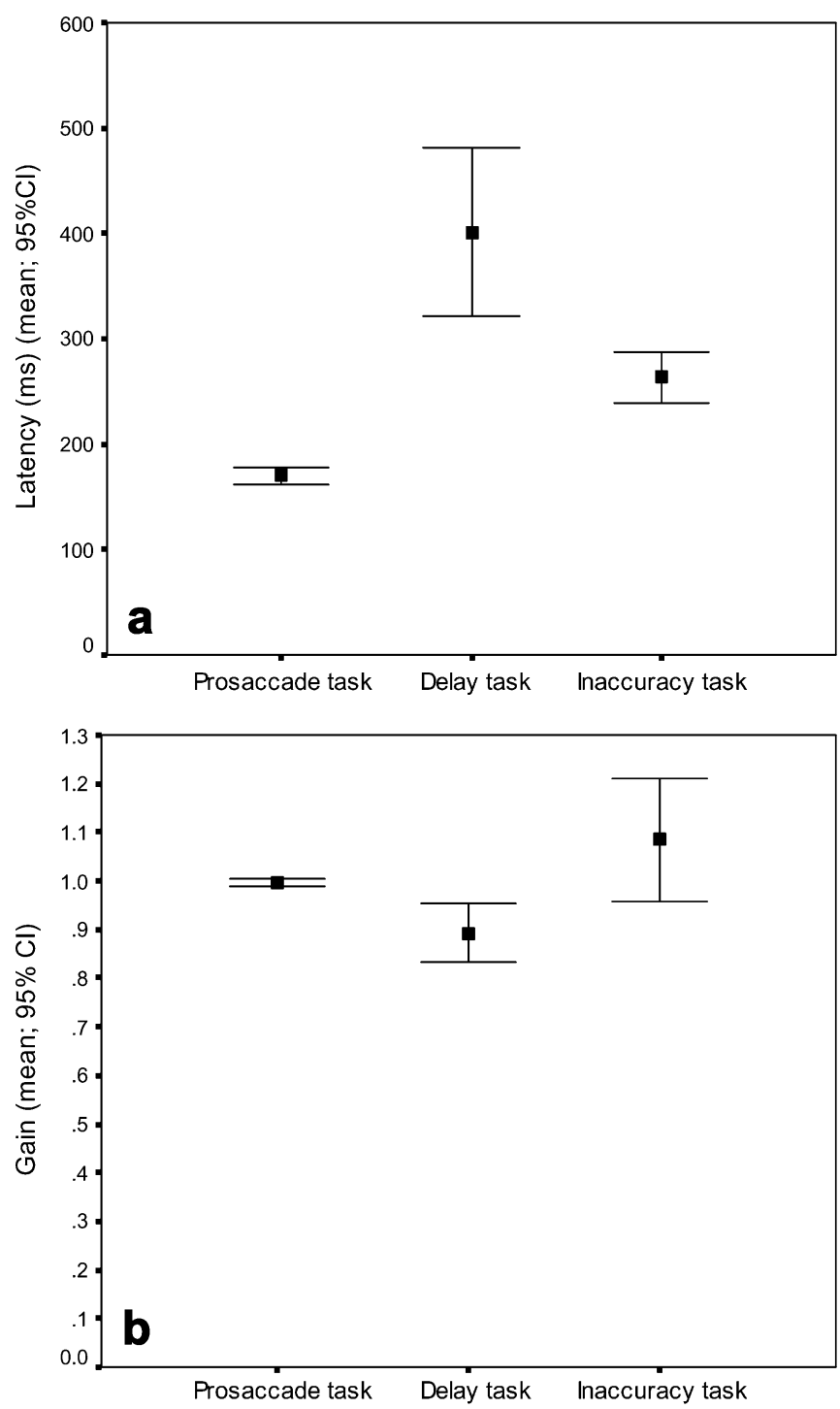

Fig. 2a, b Saccade latency and accuracy (gain) of prosaccade, delay and inaccuracy tasks for comparison. In the delay task the latency was longer and gain hypometric compared to the prosaccade task. In the inaccuracy task the gain was hypermetric and saccade latency increased compared to the prosaccade task 
Table 1 The influence of volition on the standard deviation (SD). Values are the means of the individual SDs; in brackets the SD of the SD (gain $=$ saccade amplitude/target amplitude)

\begin{tabular}{llll}
\hline & Prosaccade task & Delay task & Inaccuracy task \\
\hline SD latency (ms) & $37(9)$ & $154(52)^{\mathrm{a}}$ & $102(43)$ \\
SD gain & $0.17(0.09)$ & $0.21(0.07)$ & $0.36(0.18)^{\mathrm{b}}$ \\
\hline
\end{tabular}

${ }^{a}$ Independent sample $t$-test: delay vs. prosaccade task $(p<0.0001)$

${ }^{\mathrm{b}}$ Independent sample $t$-test: inaccuracy vs. prosaccade task $(p<0.0001)$

When instructed to be inaccurate $57 \%$ (SD $24 \%$ ) of the saccades were hypermetric and 39\% (SD 25\%) hypometric. Compared to regular prosaccades (Fig. 2), mean gain of inaccurate saccades was not different $(p=0.16, \mathrm{~ns})$, but associated with higher variability, i.e. SD $(p<0.0001)$ (Table 1) and increased latency $(p<0.0001)$. The latency of inaccurate saccades (mean $264 \mathrm{~ms}$; SD $53 \mathrm{~ms}$ ), however, was similar to the latency of correct antisaccades (mean $264 \mathrm{~ms}$; SD $44 \mathrm{~ms})(p=0.96, \mathrm{~ns})$.

Sixteen percent (SD 8\%) of antisaccades were erroneous antisaccades and 97\% (SD 7) were corrected. Compared to erroneous antisaccades, mean ISI of the redirection task was significantly longer $(p<0.0001)$ (Fig. $3 \mathrm{a})$ and redirected saccades were more accurate $(p=0.004)$ (Fig. 3b). The latency of erroneous antisaccades (mean $156 \mathrm{~ms}$; SD $27 \mathrm{~ms}$ ) was shorter compared to the latency of redirected saccades (mean $181 \mathrm{~ms}$; SD $28 \mathrm{~ms})(p=0.001)$ but the latter were indistinguishable from regular prosaccades $(p=0.11, \mathrm{~ns})$. Direction errors were rare in the redirection task (1.9\%) and the latency (mean $286 \mathrm{~ms}$; SD $118 \mathrm{~ms}$ ) was indistinguishable from correct antisaccades (mean $264 \mathrm{~ms}$; SD $44 \mathrm{~ms}$ ) ( $p=0.44$, ns).

\section{Discussion}

We assessed the influence of the verbal instruction on topdown saccade control and found, as expected, that saccade control is instruction dependent. Bottom-up control was a gap paradigm and was kept constant for all instructions. The instruction to delay saccade triggering was followed by longer latencies and instruction to be inaccurate led to higher gains. Present and previous findings suggest that the word-by-word content of the instruction is crucial when saccades are tested. Compared to the instruction 'to look at the target', the addition 'to look as fast as possible' or 'to be as accurate as possible' reduced saccade latency (Fischer et al. 1993) or increased accuracy (Kowler and Blaser 1995) respectively. These findings imply that whenever saccadic eye movements are tested in humans, verbal instructions should be read word-for-word in order to get reliable results.

Cognitive models propose hierarchical processing of temporo-spatial information and an overlap of bottom-up and top-down control (Findlay and Walker 1999). Whenever the instruction content regarding when and where to trigger a saccade did not match with either target position or time of target appearance, such as in the delay,
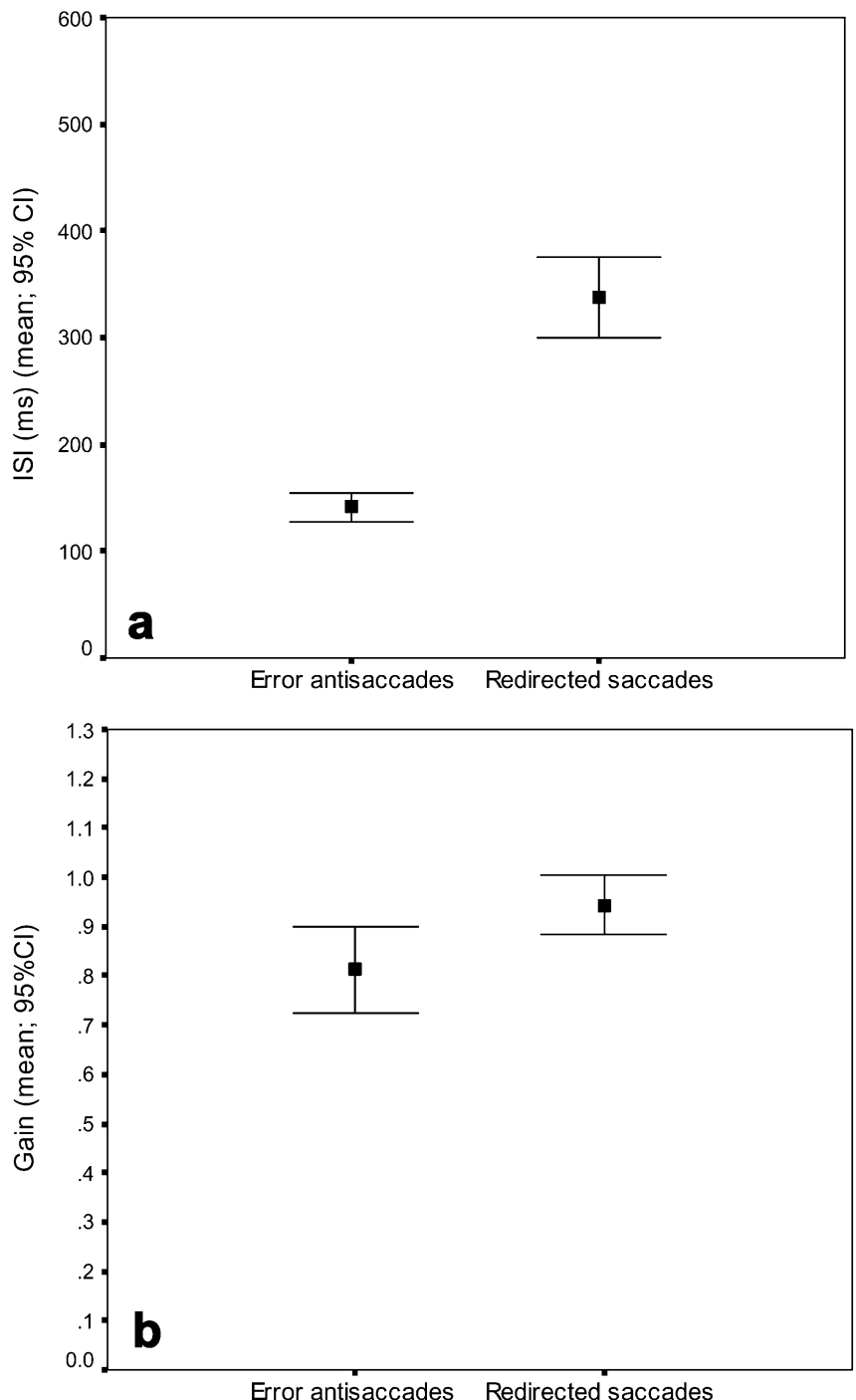

Fig. 3a, b Comparison of intersaccadic intervals (ISI) of deliberately redirected saccades and erroneous antisaccades. Compared to erroneous antisaccades deliberately redirected saccades had longer ISIs and higher gain

inaccuracy and antisaccade tasks, top-down control was required for conflict resolution, i.e. for the suppression, the redirection, or the cognitive modification of the temporospatial parameters of saccades.

The dorsolateral prefrontal cortex is important for saccade suppression and anatomical studies suggest an inhibitory fronto-collicular projection (Pierrot-Deseilligny et al. 2003a). Saccade suppression was associated with errors, i.e. the suppression of bottom-up driven visuallyguided saccades failed. This observation is in agreement with previous studies reporting errors in suppressing bottom-up driven reflexive saccades in healthy controls (Everling and Fischer 1998). Given that about $100 \mathrm{~ms}$ are required to perceive a visual stimulus in humans and that bottom-up driven saccades are triggered in the range of $120-180 \mathrm{~ms}$, the time for top-down interference is short. Whenever this slot is missed bottom-up control seemed to predominate. 
Top-down processing was needed for the spatial redirection of saccades as in the antisaccade and inaccuracy tasks. In these tasks saccade triggering had to be halted until the spatial parameters were modified. The latencies of the inaccuracy and antisaccade tasks were similar but longer compared to regular prosaccades, although saccades were directed towards the targets in the inaccuracy task and opposite to the targets in the antisaccade task. Latencies in the inaccuracy and antisaccade tasks are therefore likely to reflect saccade suppression and spatial reprogramming (Fischer and Weber 1992), rather than shifting spatial target representation from the contralateral to the ipsilateral hemisphere (Hallett 1978).

When instructed to delay saccades and to make inaccurate saccades, latency and accuracy were detrimentally affected and associated with higher variability. Cognitive models have suggested separate but interconnected pathways for temporo-spatial information processing (Findlay and Walker 1999) in basal ganglia (Hikosaka et al. 2000; Munoz et al. 2000) and cortex (PierrotDeseilligny et al. 2003b), but when instructed to delay or to be inaccurate, subjects were unable to control either spatial or temporal parameters solely. Optimal performance was only achieved when saccades were bottom-up controlled, whenever top-down control was predominating, latencies got longer or amplitudes less accurate.

Compared to erroneous antisaccades, the longer intersaccadic interval (ISI) required to redirect saccades in the redirection task brings into question whether parallel processing is possible during top-down control. Although subjects could anticipate that the second saccade will be redirected opposite to the targets, the first saccade towards the targets was bottom-up controlled and the corrective saccade was an intentional saccade. This is in agreement with the findings by Mokler and Fischer (1999), who showed that the time required to trigger a corrective saccade depended on the realisation of the direction error in the antisaccade task and longest correction times were found, when the sequence was made volitionally. In this study an ISI of $145 \mathrm{~ms}$ has been suggested for realised and $95 \mathrm{~ms}$ for unrealised errors. Although we did not ask the volunteers for feedback in the antisaccade task, the ISI found in the present study for erroneous antisaccades (140 $\mathrm{ms}$ ) and the high error correction rate suggest that most of the subjects have realised their errors. Other studies (Inhoff 1986; Zingale and Kowler 1987) found that the latency of the first saccade increased with the number of forthcoming targets, when a sequence of targets was presented. These studies presented multiple targets, whereas in the present study only one stimulus was presented and second saccade in the sequence was therefore not bottom-up controlled. Taken together, these findings suggest sequential processing for top-down control and predominance of parallel processing in bottom-up control (Becker and Jurgens 1979).

Few studies have previously addressed the impact of the instruction content. Top-down control is a time consuming process interfering with both temporal and spatial parameters of saccade genesis. It is associated with higher variability of the temporo-spatial parameters and is susceptible to errors i.e. missed suppression of bottomup control. It is questionable whether parallel processing is possible for top-down control. Hopefully this investigation will stimulate research focussing on the interaction between instruction and saccade performance.

\section{References}

Becker W, Jurgens R (1979) An analysis of the saccadic system by means of double step stimuli. Vision Res 19:967-983

Everling S, Fischer B (1998) The antisaccade: a review of basic research and clinical studies. Neuropsychologia 36:885-899

Findlay JM, Walker R (1999) A model of saccade generation based on parallel processing and competitive inhibition. Behav Brain Sci 22:661-674; discussion 674-721

Fischer B, Weber H (1992) Characteristics of "anti" saccades in man. Exp Brain Res 89:415-424

Fischer B, Weber H, Biscaldi M, Aiple F, Otto P, Stuhr V (1993) Separate populations of visually guided saccades in humans: reaction times and amplitudes. Exp Brain Res 92:528-541

Hallett PE (1978) Primary and secondary saccades to goals defined by instructions. Vision Res 18:1279-1296

Hikosaka O, Takikawa Y, Kawagoe R (2000) Role of the basal ganglia in the control of purposive saccadic eye movements. Physiol Rev 80:953-978

Inhoff AW (1986) Preparing sequences of saccades under choice reaction conditions: effects of sequence length and context. Acta Psychol (Amst) 61:211-228

Kowler E, Blaser E (1995) The accuracy and precision of saccades to small and large targets. Vision Res 35:1741-1754

Leigh RJ, Kennard C (2004) Using saccades as a research tool in the clinical neurosciences. Brain 127:460-477

Mokler A, Fischer B (1999) The recognition and correction of involuntary prosaccades in an antisaccade task. Exp Brain Res 125:511-516

Munoz DP, Dorris MC, Pare M, Everling S (2000) On your mark, get set: brainstem circuitry underlying saccadic initiation. Can J Physiol Pharmacol 78:934-944

Pierrot-Deseilligny C, Muri RM, Ploner CJ, Gaymard B, Demeret S, Rivaud-Pechoux S (2003a) Decisional role of the dorsolateral prefrontal cortex in ocular motor behaviour. Brain 126:1460 1473

Pierrot-Deseilligny C, Muri RM, Ploner CJ, Gaymard B, RivaudPechoux S (2003b) Cortical control of ocular saccades in humans: a model for motricity. Prog Brain Res 142:3-17

Turner M (1997) Malingering. Br J Psychiatry 171:409-411

Zingale CM, Kowler E (1987) Planning sequences of saccades. Vision Res 27:1327-1341 